\title{
Normal Modes and Modal Reduction in Exterior Acoustics
}

\author{
Lennart Moheit* and Steffen Marburg \\ Chair of Vibroacoustics of Vehicles and Machines \\ Department of Mechanical Engineering \\ Technical University of Munich, Garching 85748, Germany \\ *lennart.moheit@tum.de
}

Received 31 December 2017

Accepted 29 March 2018

Published 14 June 2018

\begin{abstract}
The Helmholtz equation for exterior acoustic problems can be solved by the finite element method in combination with conjugated infinite elements. Both provide frequency-independent system matrices, forming a discrete, linear system of equations. The homogenous system can be understood as a quadratic eigenvalue problem of normal modes (NMs). Knowledge about the only relevant NMs, which - when doing modal superposition - still provide a sufficiently accurate solution for the sound pressure and sound power in comparison to the full set of modes, leads to reduced computational effort. Properties of NMs and criteria of modal reduction are discussed in this work.
\end{abstract}

Keywords: Exterior acoustics; infinite element method; normal modes; modal reduction.

\section{Introduction}

Exterior acoustic problems comprehend the propagation and distribution of sound pressure in fluid-filled domains of infinite extent. This implies sound sources under free-field conditions in full- or half-space problems, with or without open cavities. For the description of sound sources, e.g. by means of their radiated sound power, free-field conditions are required in order to only determine the characteristics of the source and to exclude the influence of the measurement environment. This also applies for the numerical simulation of sound radiation, since in the context of virtual prototyping, it is desired for estimating acoustical properties in the development process. For this purpose, the Sommerfeld radiation condition ${ }^{1}$ has to be satisfied, according to which the sound pressure decays with a defined rate and vanishes towards infinity.

The classical finite element method $(\mathrm{FEM})^{2}$ is restricted to interior acoustic problems in enclosed computational domains with reflecting or partially absorbing boundary conditions (ABCs). Givoli et al $!^{34}$ introduce and review the existing high-order local ABCs with the aim of applying the FEM to exterior acoustic problems. Rabinovich et al! 5 compare the

This is an Open Access article published by World Scientific Publishing Company. It is distributed under the terms of the Creative Commons Attribution 4.0 (CC-BY) License. Further distribution of this work is permitted, provided the original work is properly cited. 
high-order ABCs with perfectly matched layers (PMLs) that were introduced by Berenger ${ }^{6}$ and optimized for frequency domain problems by Bermúdez et al ${ }^{78}$ PMLs ensure nonreflective outer boundaries of the computational domain by attaching additional damped and finite computational domains to its outer boundary with perfect impedance matching at the junction. Similar to the PML approach, the infinite element method (IFEM) works with an outer layer around an inner spherical or ellipsoidal FE domain. Different formulations have been presented, e.g. by Bettess, $\frac{9}{9}$ Burnett $\frac{10}{10}$ and Astley ${ }^{11}$ The Astley-Leis IFEM formulation provides frequency-independent system matrices, ${ }^{[2] 13}$ which is required for the subsequent investigations in this work. The radial interpolation of the sound pressure between the outer FE boundary and infinity can be realized by different polynomials. Using Legendre or Jacobi polynomials leads to improved matrix conditioning in comparison to conventional Lagrange polynomials, according to von Estorff and Dreyer et al.14]15

The Sommerfeld radiation condition is also implied in the boundary element method (BEM) $\frac{16[17}{17}$ where an integral equation is found to solve the Helmholtz equation at the surface of the sound source for the given set of boundary conditions. This leads to discrete and frequency-dependent, single-layer and double-layer potential matrices that associate the sound pressure and fluid particle velocity at the boundary.

In order to determine the sound pressure or sound power using one of the above methods, linear systems of equations have to be solved and matrices have to be inverted for each frequency of interest separately. This leads to considerable computational effort for studies with a large number of degrees of freedom (DOFs) in a wide frequency range. A possible model reduction approach is modal analysis and superposition, in which the solution is decomposed into modes — or, in mathematical terms, eigenvalues and eigenvectors — which describe theoretically possible and orthogonal shapes of vibration that can be summed up or superimposed to the total solution.

The concept of normal mode (NM) was first adapted to exterior acoustic problems by Marburg et al. $\frac{18}{20}$ and further investigated by Moheit and Marburg. 2122 They apply the frequency-independent Astley-Leis IFEM in order to solve a single linearized eigenvalue problem referring to the works by Ruge 23 and Tisseur and Meerbergen.24 The authors investigate the influence of the mesh and the radial interpolation of the IFEM on NMs and acoustic radiation modes (ARMs) and compare both kinds of modes in exterior acoustic problems. Fuß et al. ${ }^{25}$ use an Arnoldi eigenvalue solver to calculate selected, weakly damped NMs in a proximity to the imaginary axis iteratively. The modal reduction of ARMs has been investigated by Kuijpers et al., ${ }^{[6]}$ Kessels ${ }^{27}$ and Peters et al. 28$] 30$ using BEM.

Model reduction is mainly associated with Krylov subspace-based methods such as Padévia-Lanczos and Padé-via-Arnoldi. The latter approach has been applied to fluid-loaded structural modes by Peters et al. in Ref. 30 using a fully-coupled FEM/BEM model, whereas Baugart et al. $\stackrel{31}{31}$ apply the Padé-via-Lanczos algorithm to FE- and IFE-discretized exterior problems in order to predict sound power efficiently. Wagner et al ${ }^{32}$ describe the concept of the Krylov subspace approach on the basis of Dirichlet-to-Neumann (DtN) boundary conditions and IFEs in exterior acoustics with the aim of solving the Helmholtz equation simultaneously at multiple frequencies. 
In this phenomenological work, the authors recapitulate the NMs approach and the determination of the sound pressure and sound power and investigate the properties of eigenvalues and eigenvectors for different geometries and load cases. In particular, the distinction between pure exterior problems and cavity problems is worked out. Finally, the errors of the radiated sound power are determined for reduced modal bases with certain criteria of modal reduction.

\section{Method}

According to the work by Marburg et al., 18 20 $\mathrm{NMs}$ in numerical exterior acoustic problems can be determined as follows: For the description of the spatial sound pressure field $p(\mathbf{x})$ at a frequency $f$, the Helmholtz differential equation is used. It is discretized by the FEM and the IFEM according to Astley and Leis. The general setup is depicted in Fig. 1.

The resulting discrete system of $N$ linear equations $-\omega^{2} \mathbf{M}-i \omega \mathbf{D}+\mathbf{K}=\mathbf{f}$ is solved as a linearized quadratic eigenvalue problem with $2 N \times 2 N$-sized hypermatrices $\mathbf{A}$ and $\mathbf{B}$ :

$$
\mathbf{A}=\left[\begin{array}{cc}
\mathbf{M} & \mathbf{0} \\
\mathbf{0} & -\mathbf{K}
\end{array}\right], \quad \mathbf{B}=\left[\begin{array}{cc}
\mathbf{0} & \mathbf{M} \\
\mathbf{M} & \mathbf{D}
\end{array}\right] .
$$

This leads to $2 N$ eigenvalues $\kappa_{m}$ corresponding to left and right eigenvectors $\mathbf{y}_{z, m}$ and $\mathbf{x}_{z, m}$ due to nonsymmetric system matrices for the IFEs

$$
\left(\mathbf{A}-\kappa_{m} \mathbf{B}\right) \mathbf{x}_{z, m}=\mathbf{0} \quad \text { and } \quad \mathbf{y}_{z, m}^{T}\left(\mathbf{A}-\kappa_{m} \mathbf{B}\right)=\mathbf{0} .
$$

The subscript $z$ indicates the twofold length of the eigenvectors as a consequence of the statespace linearization. The eigenvectors can be column-wisely comprised in modal matrices, which are indicated by the capital letters in what follows. The orthogonality of the modes is measured by the product of modal matrices and hypermatrices

$$
\mathbf{Y}_{z}^{T} \mathbf{A X}_{z}=\operatorname{diag}\left(\alpha_{1}, \ldots, \alpha_{2 N-\delta}\right) \quad \text { and } \quad \mathbf{Y}_{z}^{T} \mathbf{B X}_{z}=\operatorname{diag}\left(\beta_{1}, \ldots, \beta_{2 N-\delta}\right),
$$

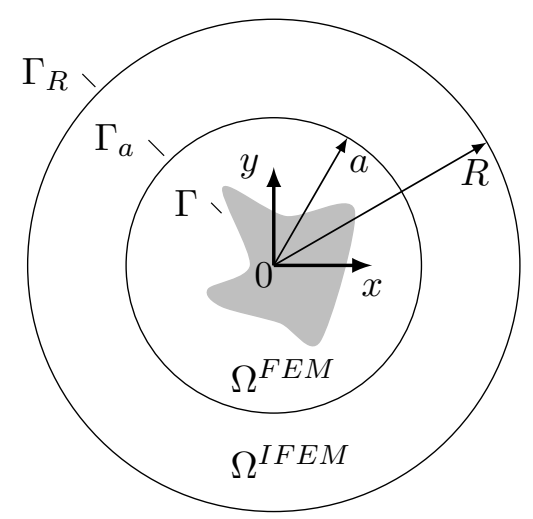

Fig. 1. General setup of the FEM/IFEM discretization: sound source as an obstacle (gray) in the fluid-filled computational domains $\Omega^{\mathrm{FEM}}$ (radius $a$ ) and $\Omega^{\mathrm{IFEM}}$ (outer radius $R$ ). 


\section{Moheit \&S S. Marburg}

where the ratio of the entries on the diagonals is found as the eigenvalues $\kappa_{m}=\alpha_{m} / \beta_{m}$. The eigenvalues are complex numbers and their real part contains information about the damping of the respective mode, whereas their imaginary part indicates its resonance frequency. $\delta$ is the rank deficiency of the mass matrix. A circular shape of the FE domain leads to an empty mass matrix for all DOFs of the IFEs. ${ }^{[2]}$ Marburg ${ }^{20}$ suggested canceling empty rows and columns, which leads to a system of equations of reduced size $2 N-\delta$.

It is notable that only one single eigenvalue problem has to be solved, since the system matrices do not depend on the frequency and neither do the eigenvalues and eigenvectors.

\subsection{Modal sound pressure and sound power}

Up to this point, the eigensolution of the problem is frequency-independent and does not consider any right-hand-side excitation $\mathbf{f}$, e.g. caused by a structural particle velocity $v_{s}(\mathbf{x})$ at the boundary of inner obstacles. The discrete sound pressure field can be constructed by modal superposition of the truncated eigenvectors $\mathbf{y}_{m}$ and $\mathbf{x}_{m}$ of size $N \times 1$, considering only those $N$ DOFs that are related to the pressure as shown by Marburg in Ref. 20.

$$
\mathbf{p}=-\sum_{m=1}^{2 N-\delta} \frac{\mathbf{y}_{\Gamma, m}^{T} \mathbf{f}_{\Gamma}}{\alpha_{m}+i k \beta_{m}} \mathbf{x}_{m}
$$

where the wave number $k=\omega / c_{f}$ is the ratio of the angular frequency $2 \pi f$ and the speed of sound of the fluid. The index $\Gamma$ indicates that only those DOFs in the right-hand-side vector and in the eigenvectors have to be taken into account that are related to the surface of the inner obstacles. The modal basis can be reduced if the sum is formed by the given eigenvectors and eigenvalues. In the same manner, the radiated sound power can be superimposed by a reduced number of modes. The discrete definition of the radiated sound power includes the discrete sound pressure and the particle velocities at the radiating boundaries 2028 .

$$
P=\Re\left\{\frac{1}{2} \mathbf{p}_{\Gamma}^{T} \Theta \mathbf{v}_{\Gamma}^{*}\right\},
$$

where $\Theta$ is the boundary mass matrix ${ }^{[17}$ Hence, the sound power $P_{\mathrm{NM}}$ is found as the sum of modal sound power distributions $P_{m}$ with

$$
P_{\mathrm{NM}}=\Re\left\{-0.5 \frac{1}{i \omega \rho_{f}} \sum_{m=1}^{2 N-\delta} \frac{\mathbf{y}_{\Gamma, m}^{T} \mathbf{f}_{\Gamma}}{\alpha_{m}+i k \beta_{m}} \mathbf{x}_{\Gamma, m}^{T} \mathbf{f}_{\Gamma}^{*}\right\} .
$$

The sound power $P$ is called $P_{\text {Ainv }}$ in what follows, as long as the included sound pressure vector in Eq. (5) is obtained by full inversion of the dynamic stiffness matrix $\mathbf{A}$ according to

$$
\mathbf{p}_{\Gamma}=\left[\mathbf{A}^{-1} \mathbf{f}\right]_{\Gamma}=\left[\left(-\omega^{2} \mathbf{M}-i \omega \mathbf{D}+\mathbf{K}\right)^{-1}\left(-i \omega \rho_{f} \Theta \mathbf{v}_{s}\right)\right]_{\Gamma} .
$$

\section{Models and Implementation}

Three geometries are modeled and the respective FE matrices are computed by using the commercial software COMSOL Multiphysics ${ }^{\circledR}$. The matrices as well as the mesh information 
are exported to MATLAB, where the subsequent processing is done. This includes the implementation of the IFEM, the computation of the NMs and the radiated sound power as well as the visualization of the results. All models are two-dimensional problems that consider a solid structure as a sound-hard obstacle in a circular, fluid-filled FE domain, where the IFEs are attached to the outer boundary of the FE mesh according to the setup in Fig. 11.

The first model (a) is an ellipse-like structure with a rectangular insertion over the whole length of the ellipsoidal obstacle along the $x$-axis with a height of $0.1 \mathrm{~m}$. The two semi-axes of the halfway stretched ellipses are $a=0.9 \mathrm{~m}$ and $b=17 / 30 \mathrm{~m} \approx 0.57 \mathrm{~m}$. The second geometry (b) is a slight modification of the first one. Here, a part of the inner rectangular insertion is cut out at the right side with a length of $1.7 \mathrm{~m}$. The third obstacle (c) is the inversion of the second geometry, where the ellipse is removed and only the former cavity is an inner obstacle in the fluid domain. The geometries and meshes are depicted in Fig. 2.

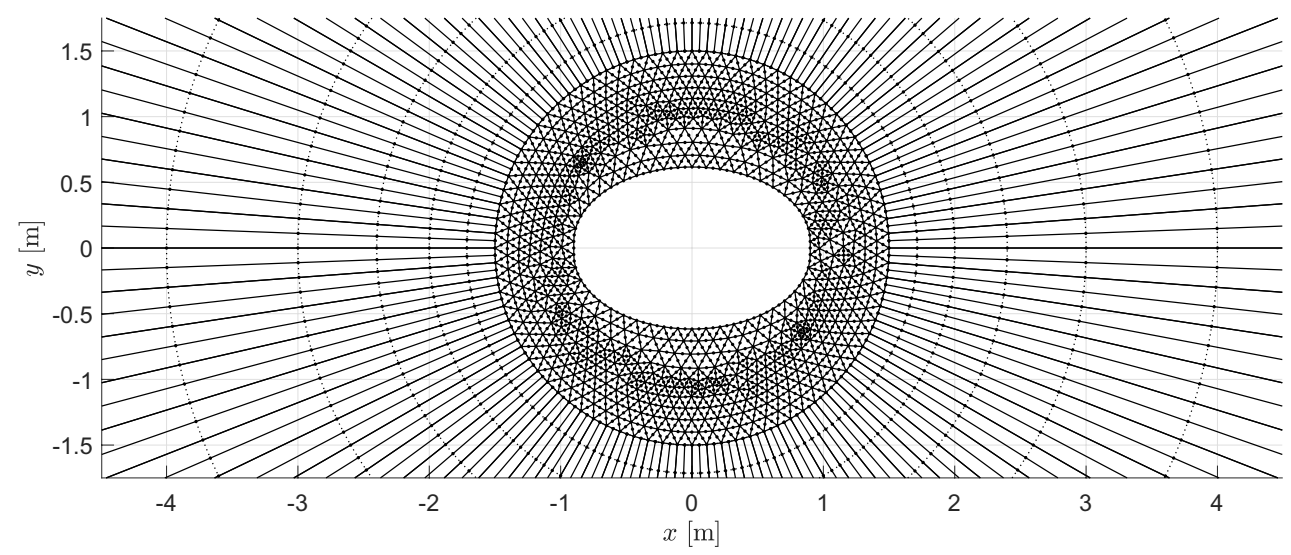

(a) Ellipsoidal obstacle

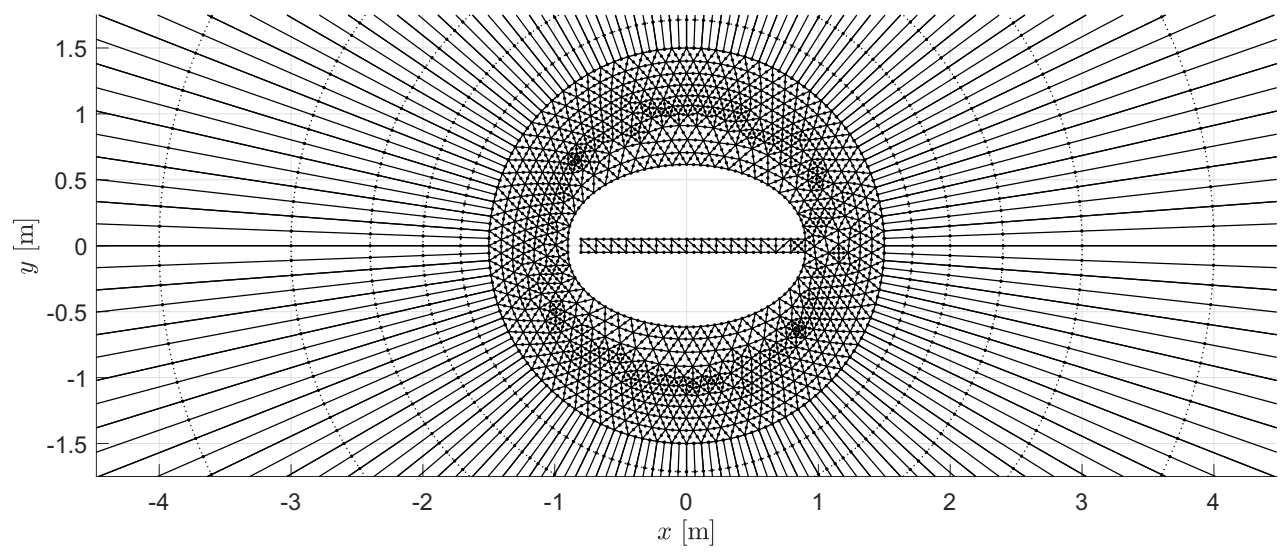

(b) Ellipsoidal obstacle with rectangular cavity

Fig. 2. Meshes in the fluid-filled domain around the three inner obstacles discretized by FE and IFEs. 


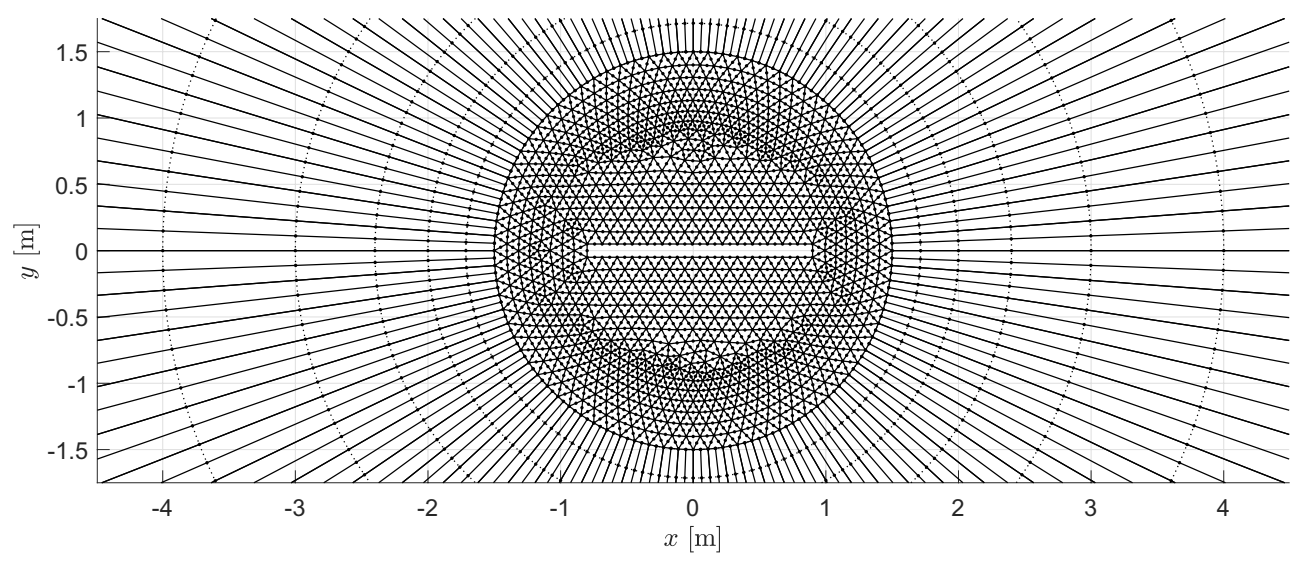

(c) Rectangular obstacle

Fig. 2. (Continued)

The three chosen geometries are motivated as follows: The fluid in the computational domain is air with a density of $\rho_{f}=1.3 \mathrm{~kg} \mathrm{~m}^{-3}$ and a speed of sound of $c_{f}=340 \mathrm{~ms}^{-1}$. In the case of a closed tube with a length of $1.7 \mathrm{~m}$, the undamped eigenvalue problem leads to eigenfrequencies in $100 \mathrm{~Hz}$-steps, starting from the first natural frequency at $0 \mathrm{~Hz}$ and corresponding to standing waves along the long side of the tube. In the case of the open cavity in the exterior acoustic domain, the eigenfrequencies are expected to appear damped at lower frequencies, but still can be found with roughly the same frequency step from one eigenfrequency to the next. This helps to better identify cavity-related resonance frequencies in the solution. In comparison to the ellipsoidal obstacle with the duct-like cut-out (b) and with these expected cavity modes inside, the ellipsoid (a) and the rectangle (c) are pure exterior problems. They all have the same IFE discretization and only differ in the inner area of the FE domain close to the surface of the obstacles, which might reveal similarities and differences in the spectral solution. In these examples, the role of pure real and complex eigenvalues and eigenvectors with respect to cavity-related resonances and outer multipole modes shall be investigated.

All surfaces of the obstacles behave reverberantly in such a way that the boundary admittance is zero $Y(\mathbf{x})=0$ and the particle velocities at the surfaces are the same in the fluid and in the structure $v_{f}=v_{s} \cdot 17$ The FE mesh has a maximum element size of $h_{\max }=11.33 \mathrm{~cm}$ in order to ensure at least six elements per wavelength at a frequency of $500 \mathrm{~Hz}$ for second-order Lagrangian FEs, which seems to be an appropriate sampling according to the literature Refs. 33 and 34 .

At each of the 84 quadratic line elements at the outer circular FE boundary, an IFE is attached. Its polynomial order for the interpolation in the transversal direction is inherited from the corresponding boundary line. For the interpolation in the radial direction, the authors used 8-order Jacobi polynomials with the two corresponding exponents $\alpha=1$ and $\beta=0$. According to von Estorff et al.$^{14}$ and Dreyer et al. ${ }^{[15}$ Jacobi polynomials provide a much better matrix condition number of the system matrices in comparison to Lagrange 
Table 1. Number of DOFs of the three geometries.

\begin{tabular}{lccc}
\hline Geometry & DOF FE & DOF IFE & DOF Total \\
\hline Ellipsoid (a) & 2746 & 1176 & 3922 \\
Ellipsoid with cut-out (b) & 2840 & 1176 & 4016 \\
Rectangle (c) & 3404 & 1176 & 4580 \\
\hline
\end{tabular}

polynomials. This observation was confirmed by the authors in Ref. 21 however, the influence on NMs and ARMs was negligible. The number of DOFs in the used meshes is given in Table 1

Two different load cases are investigated. In the first case, a structural particle velocity $v_{s, \text { in }}=1 \mathrm{~ms}^{-1}$ is applied to the inner left surface of the open duct. The other case is considered for two geometries, the ellipsoid and the ellipsoid with the cavity. A structural particle velocity $v_{s, \text { out }}=1 \mathrm{~ms}^{-1}$ is applied to all outer surfaces of the ellipsoid without the right surface of the rectangular insertion. Both excitations apply for all frequencies from $1 \mathrm{~Hz}$ to $500 \mathrm{~Hz}$ in $1 \mathrm{~Hz}$-steps.

Reference solutions of the radiated sound power were computed by using COMSOL Multiphysics ${ }^{\circledR}$ and by applying a circular PML around the inner FE domain with a much finer mesh. The computation time of the presented method is not yet competitive to commercial codes, since it is not optimized for performance and only efficient if a reduced number of modes is considered during the modal superposition. Currently, the whole modal basis is computed in order to investigate the results regarding their characteristics and relevance and makes the approach hardly comparable to the PML approach, where the resulting radiated sound power is determined directly and efficiently.

\section{Results}

\subsection{Eigenvectors}

The NM eigenvectors are found in the whole computational fluid domain including FE and IFE DOFs. However, in general, the main focus is on the sound pressure distribution at the mesh nodes close to the inner obstacles, i.e. in the FE domain. Three typical mode shapes are column-wisely depicted in Fig. 3 in the example of the ellipsoidal with the open duct. The authors distinguish between modes with the sound pressure mainly concentrated at or close to the surface of the inner obstacles or inside a cavity, those with the sound pressure mainly concentrated at the junction between the FE and IFE domains and, finally, those modes with an even distribution.

In order to distinguish between these three kinds of mode shapes, the sum of certain eigenvector entries is set in relation to the sum of the remaining entries associated to the DOFs in the FE domain. In doing so, the concentration of the sound pressure - either at the surface of the inner obstacles or at the junction of the FE and the IFE domains - can be compared to the remaining share of the sound pressure distribution in the entire fluid, and the qualitative distinction can be done automatically to a certain extent. The eigenvectors 

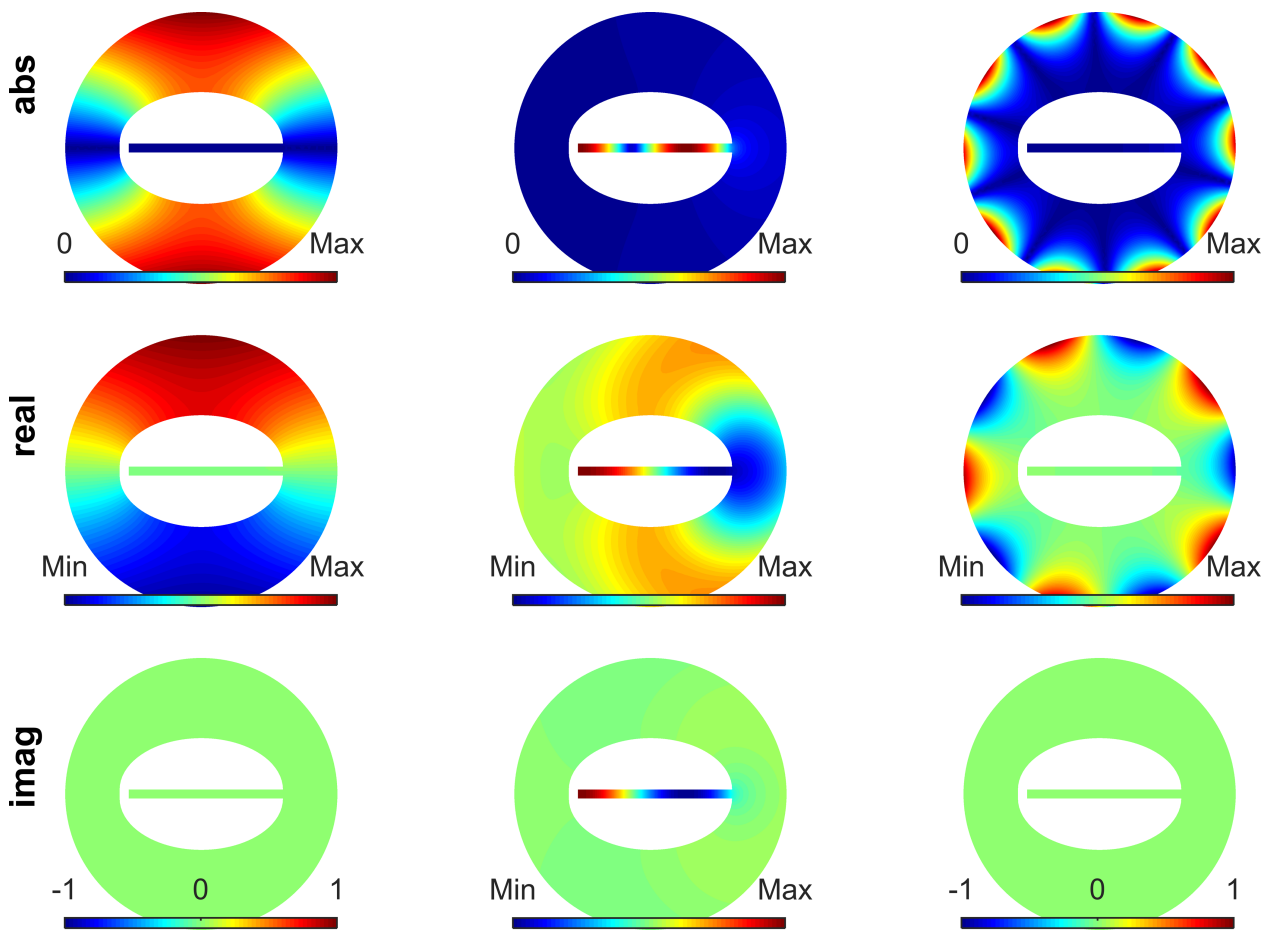

Fig. 3. Three exemplary kinds of NM shapes (one per column) as right eigenvectors $\mathbf{x}$ for the second geometry (b): even sound pressure distribution in the whole fluid domain (left); concentration of pressure peaks close to the surface of inner obstacles or cavity modes (middle); concentration of sound pressure peaks at the junction of the FE and IFE domains (right); Eigenvector magnitudes (first row), real parts (second row) and imaginary parts (bottom row).

were normalized to the length of one for this purpose and called $\hat{\mathbf{x}}$. In mathematical terms, the eigenvector distribution ratio $\chi_{m}$ for a certain mode $m$ can be written as

$$
\chi_{m}=\frac{\sum_{i}\left|\hat{\mathbf{x}}_{m, i}\right|}{\sum_{j}\left|\hat{\mathbf{x}}_{m, j}\right|} \quad \text { with } i \in \Gamma \text { and } j \in \Omega \backslash \Gamma,
$$

where $\Gamma=\Gamma_{\text {in }} \vee \Gamma_{\text {out }}$ includes either the DOFs at the inner boundaries of the obstacles $\Gamma_{\text {in }}$ or at the outer FE boundary $\Gamma_{\text {out }}$.

As mentioned in Sec. 22 the asymmetry of the IFE matrices provides left and right eigenvectors that are both part of the orthogonal modal basis and are required for the computation of the modal sound pressure and sound power contributions, see Sec. 2.1. Examples of left and right eigenvectors are visualized in Fig. 4 for IFE DOFs only, since both mode shapes are almost the same in the FE domain. The similarity of left and right eigenvectors can be measured by the modal assurance criterion (MAC), which is presented in Sec. 4.3. The left eigenvectors show a much more extended radiation pattern in the radial direction in comparison to the right eigenvectors and are in antiphase to each other. 

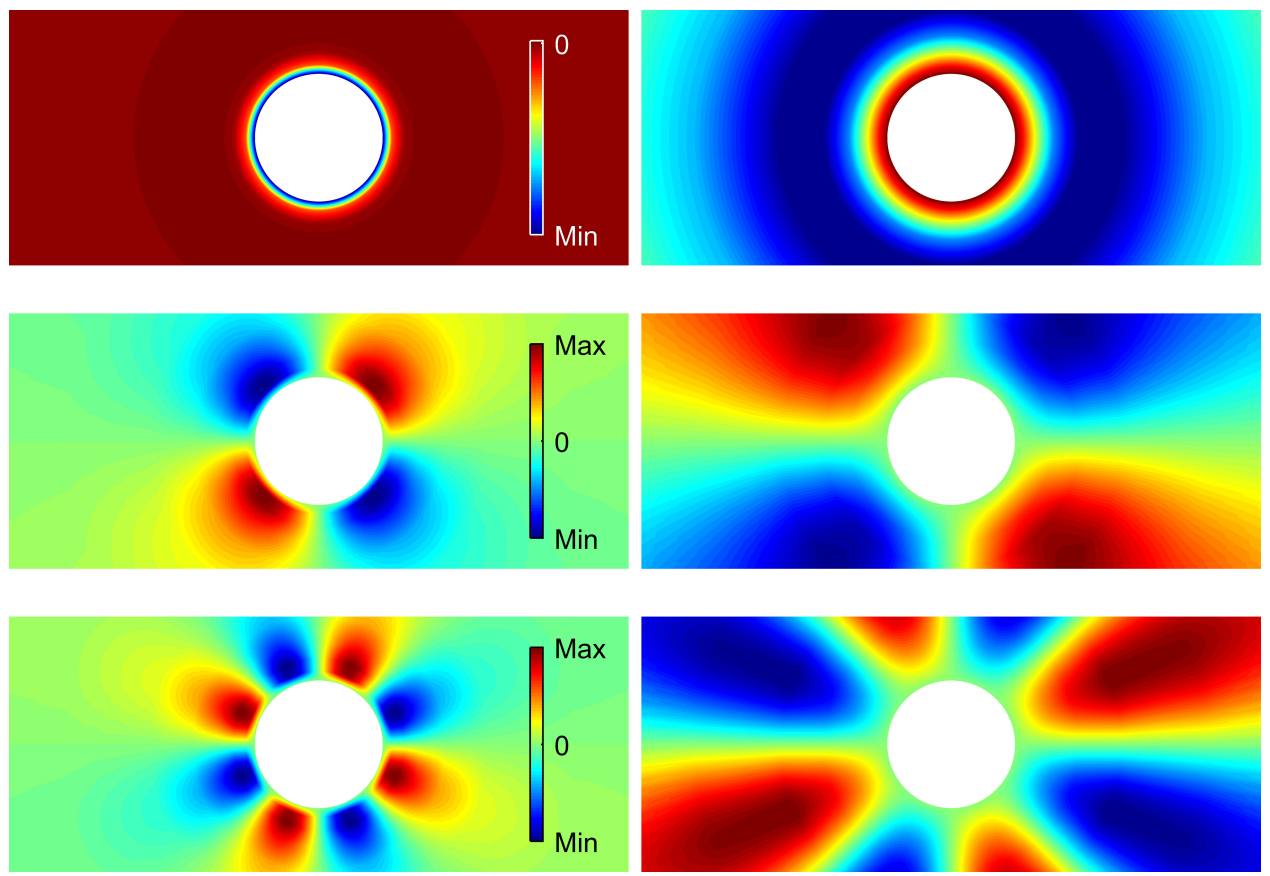

Fig. 4. Three examples of right eigenvectors $\mathbf{x}$ (left column) and the corresponding left eigenvectors $\mathbf{y}$ (right column) in the IFE domain, each both purely real and colored on the basis of the same color scale, respectively.

\subsection{Eigenvalues}

Initially, the NMs are sorted by increasing the magnitude of their eigenvalue $\kappa_{m}$. This order is not necessarily the best sequence of significance for the efficient superposition of NMs, even though weakly damped modes (small $\Re\left\{\kappa_{m}\right\}$ ) at low frequencies (small $\Im\left\{\kappa_{m}\right\}$ ) can be found in the very beginning in the list of ascending eigenvalue magnitudes. The subsequent modes might either have a weak damping or become in resonance at low frequencies in the audible frequency range, which is both an indicator of significance for the radiation of sound and therefore for the modal superposition of sound pressure or sound power. This leads to the research question of how a modified sorting algorithm could distinguish between relevant and nonrelevant modes.

The eigenvalues of the second problem (ellipse with the open duct inside) are depicted in the complex plane in Fig. 5. With each increment of the number of IFE radial interpolation points $n_{\text {rad }}$, a new straight line of highly damped eigenvalues comes up, where - at the same time - the other existing lines of eigenvalues move with a growing absolute angle in the polar form. As observed by the authors in Ref. 21, an even number for the polynomial order $n_{\text {rad }}$ leads to a line of purely real eigenvalues (cf. Fig. 5 with $n_{\text {rad }}=8$ ), whereas odd polynomial degrees do not induce these eigenvalues.

It can be observed that the eigenvalues are symmetric with respect to the real axis, which is due to the appearance of complex conjugated eigenvalues with a different sign of 

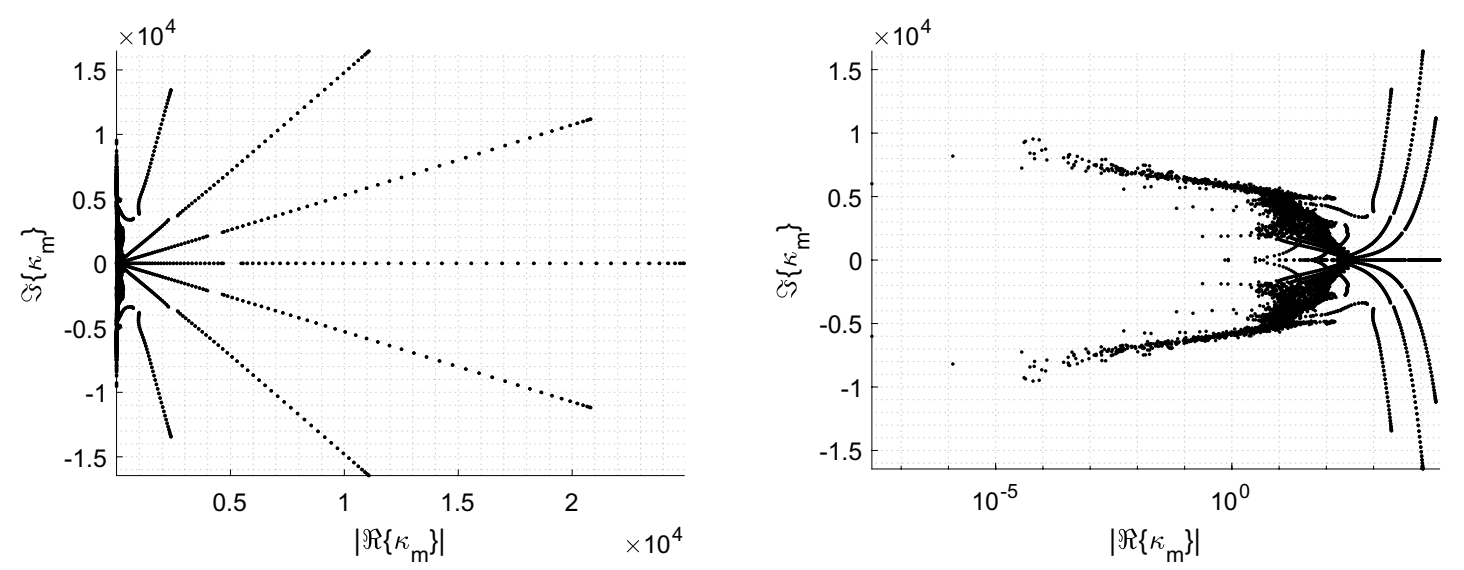

Fig. 5. NM eigenvalues of the cavity problem (second geometry) in the complex plane with linear (left) and logarithmic (right) scaling of the real axis.

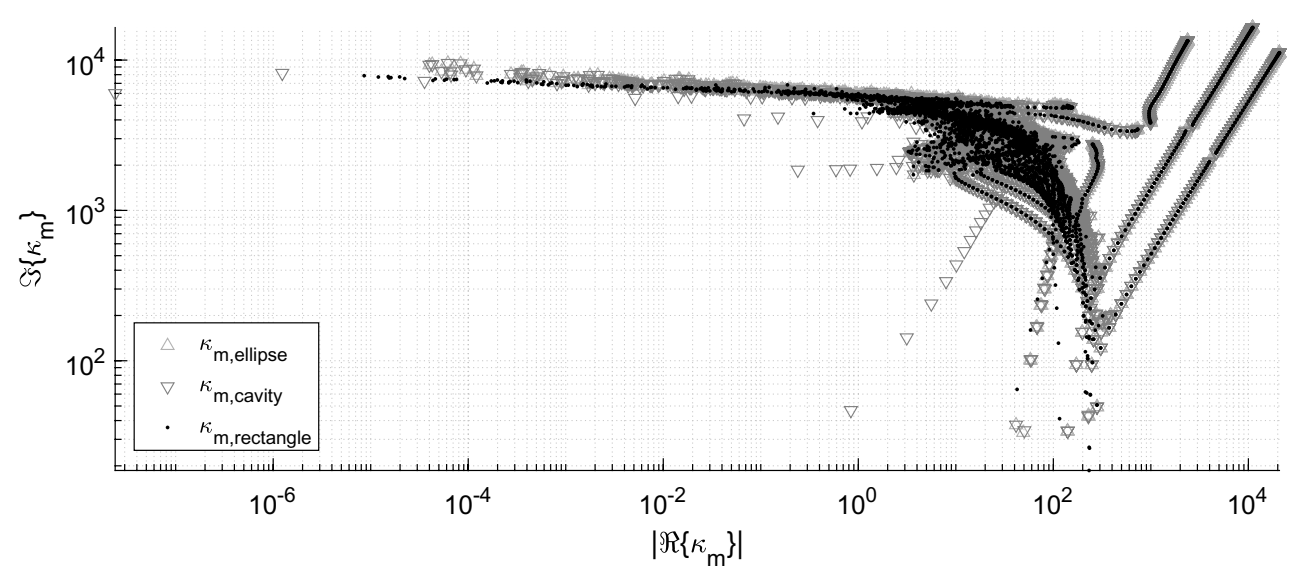

Fig. 6. Eigenvalues of the three models shown in one complex plane, considering only the positive part of the imaginary axis with double-logarithmic scaling, neglecting the purely real eigenvalues: ellipsoidal obstacle (gray deltas), ellipsoidal obstacle with the rectangular cavity (gray nablas) and rectangle (black dots).

their imaginary parts (resonance frequency), corresponding to - more or less - the same mode shape (cf. Secs. 4.1 and 4.3).

In Fig. 6] the eigenvalues of the three models are only shown in one complex plane for positive imaginary parts. In particular, most of the highly damped eigenvalues are very similar for all three problems and seem to be correlated with the properties of the IFEs. This is not the case for weakly damped eigenvalues whose positions in the complex plane seem to be more problem-dependent and therefore related to eigenvectors with a significant sound pressure distribution close to the inner obstacles. In the case of the cavity problem (b), additional lines of weakly damped eigenvalues at low frequencies can be observed. Some 
of them appear roughly in $100 \mathrm{~Hz}$-steps, which was expected by the choice of the $1.7 \mathrm{~m}$ long, duct-like cavity in the ellipse. Another line of cavity-related eigenvalues at higher frequencies begins with a weaker damping once again. These eigenvalues correspond to eigenvectors with resonances in the lateral $y$-direction of the open duct. If no inner obstacle is placed inside the FE domain, the observations can be confirmed: the highly damped eigenvalues in lines and a few multipole modes in the FE domain with small real parts of their eigenvalues can be found in the complex plane, whereas there are no problem-specific mode shapes.

According to Sec. 4.1 the eigenvectors can be roughly classified by the concentration of their pressure distribution $\chi$ at inner $\Gamma_{\text {in }}$ or outer boundaries $\Gamma_{\text {out }}$ in relation to the remaining FE DOFs in the fluid domain, see Eq. (8) and the three examples in Fig. 3. The corresponding eigenvalues can thus be rated as inner, outer or mixed modes with respect to their eigenvector distribution ratio. This was done in Fig. 7 in the example of the second geometry with the eigenvalues in the complex plane and with $\chi$ in the third dimension and additionally colored from blue to red for small to high values of $\chi$. It can be observed that the higher the real part of the eigenvalues, the higher the distribution ratio of the eigenvectors at the junction of the FE and the IFE domains (see Fig. 7(a)). The highest pressure concentration can be found for purely real eigenvalues with a real part in a range of about $10^{3}$ to $10^{4}$. On the other hand in Fig. 7(b), the highest pressure concentration at the inner obstacle is found for eigenvalues in the middle range of the real axis, where the cavity-related modes were expected in Fig. 6. The lowest concentration can be observed for highly damped modes, even though the ratios increase significantly at the very end of the real axis. In both cases, the behavior is the same for the complex conjugated partners in such a way that the lower pictures are virtually symmetric with respect to the real axis.
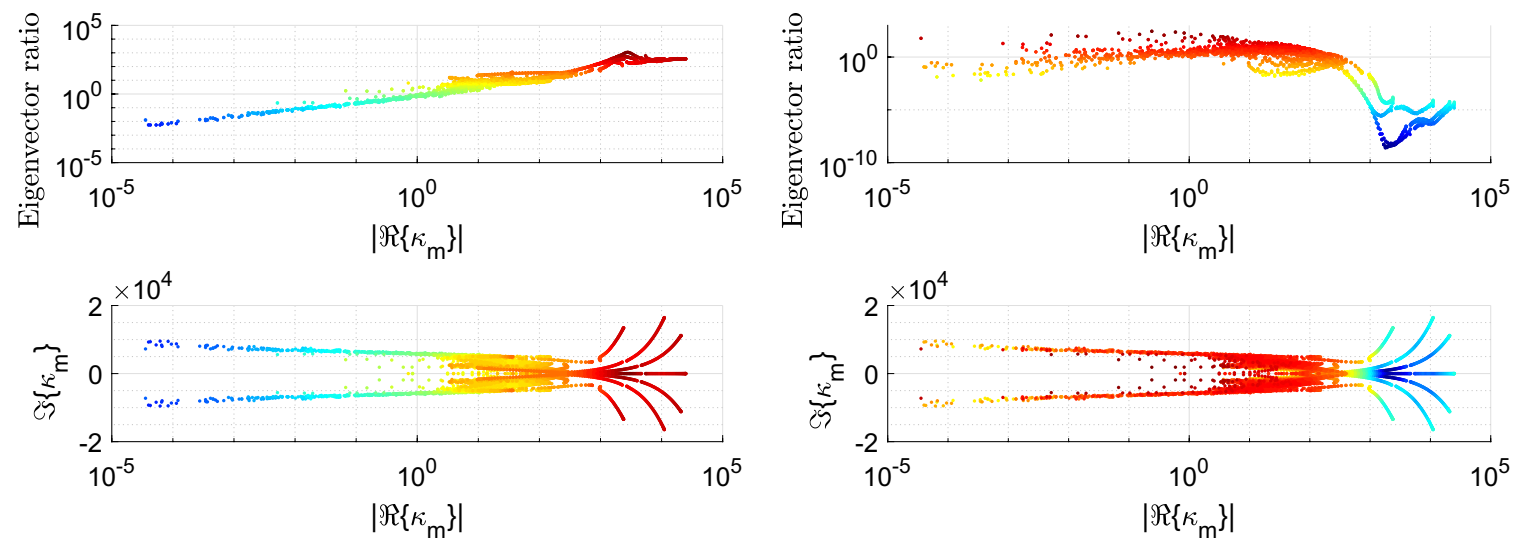

(a) Outer boundary modes

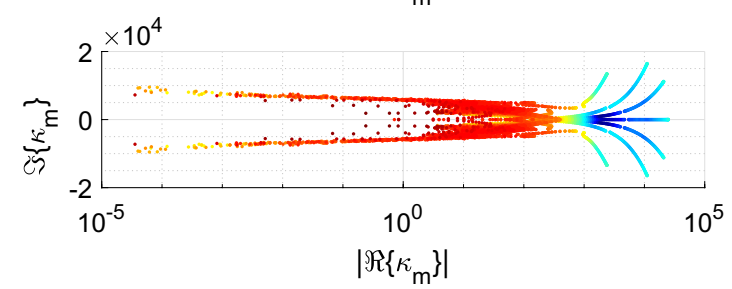

(b) Inner boundary modes

Fig. 7. (Color online) Eigenvalues in the complex plane with the ratios of the eigenvector distributions $\chi$ (see Eq. (8)) on the $z$-axis for the outer FE boundary $\Gamma_{\text {out }}$ (left) and inner obstacle boundaries $\Gamma_{\text {in }}$ (right) each with respect to the remaining DOFs; second geometry; each two views of a three-dimensional plot, where the color indicates the height on the $z$-axis (blue/small to red/high). 


\section{Moheit \&S S. Marburg}

The authors observed that purely real eigenvalues are associated with purely real eigenvectors. This applies in the same manner for complex eigenpairs.

\subsection{Modal assurance criterion (MAC)}

By means of the MAC $\stackrel{35}{5}$ the similarity of the eigenvectors to each other can be rated in an interval between zero and one. In previous work, the authors used the MAC in order to track the eigenvalues in convergence studies with different meshes and increasing orders of radial interpolation polynomials. 21

The MAC values of the left and right eigenvectors of the second geometry are depicted in Fig. 8(a). Two cases are compared to each other: either only FE DOFs (gray) or all DOFs (including the FE and IFE domains; red) are considered. Left and right eigenvectors are almost the same $(\mathrm{MAC} \approx 1$ ) according to MAC in the first case. On the other hand, only a few pairs of left and right eigenvectors are virtually identical, when all DOFs are taken into account. Here, the MAC values are distributed in almost the whole range between zero and one. In Fig. 8(b), the MAC value is added in colors (blue/zero to red/one) and to the third dimension to the complex plane of NM eigenvalues. Obviously, the similarity of left and right eigenvectors is the best for weakly damped modes, which are primarily related to large eigenvector contributions at the inner boundary lines (cf. Fig. 7(b)). Accordingly, the single peaks (with small mode numbers $m$ ) of each two modes with a relatively high MAC value for FE and IFE DOFs (red) in Fig. 8(a) are the cavity modes. For eigenvalues with a real part larger than 1 , the MAC values decrease virtually, logarithmically and with increasing damping until the values slightly grow for the very highly damped eigenvalues that do correspond to outer FE boundary modes (cf. Fig. 7(a)). The MAC values are virtually the same for both complex conjugated eigenvalue partners.

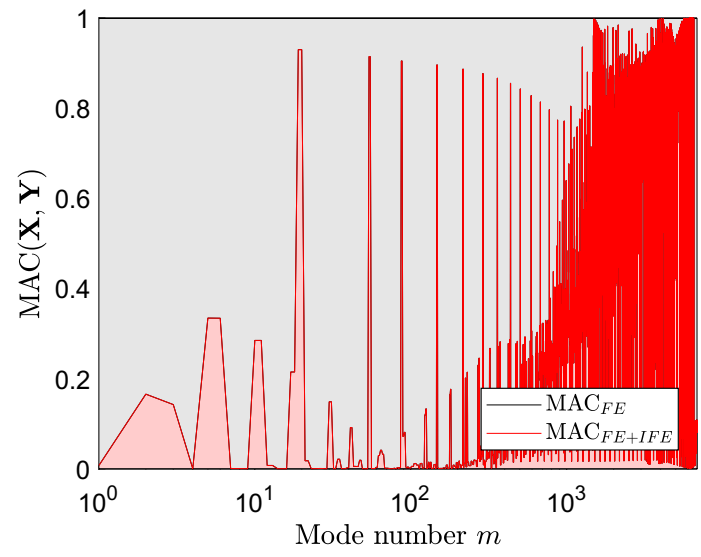

(a)

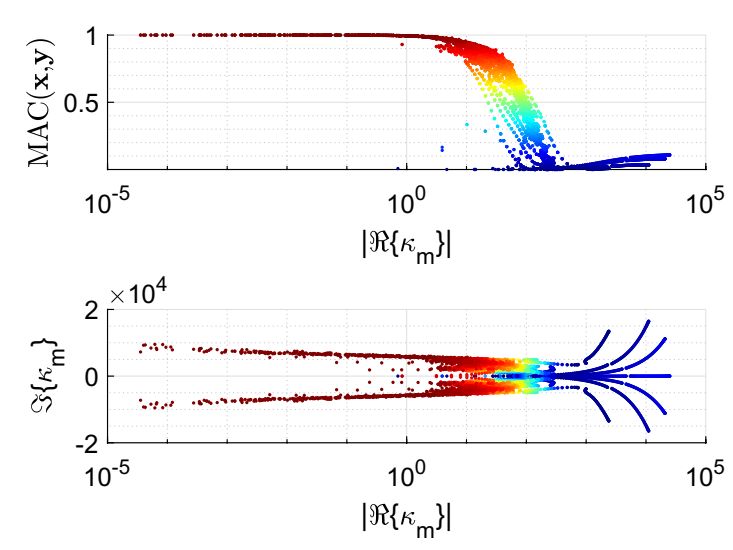

(b)

Fig. 8. (Color online) MAC of left and right NM eigenvectors in the example of the ellipse with the rectangular cavity. (a) MAC for FE DOFs only (black; almost one for all modes) and for all DOFs in the FE and the IFE domains (red) and (b) Complex plane of NM eigenvalues $\kappa_{m}$ with the MAC value in the third dimension and colored from blue/zero to red/one. 


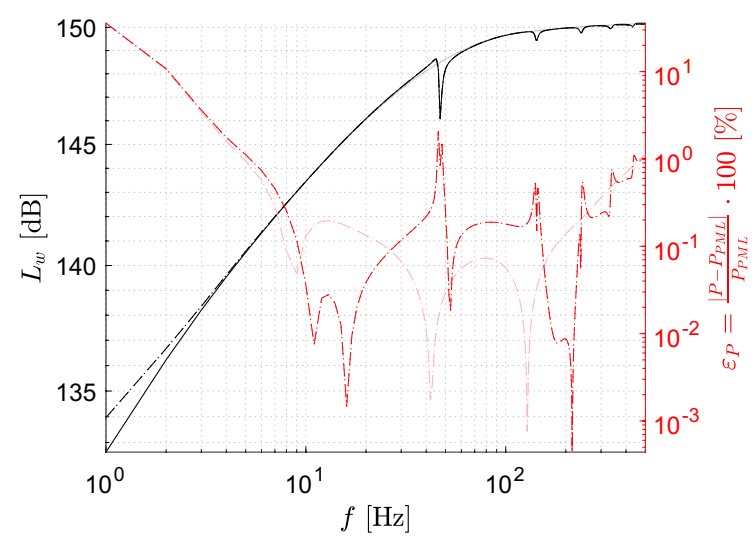

(a) $v_{s, \text { out }}=1 \mathrm{~ms}^{-1}$ geometries (a) and (b).

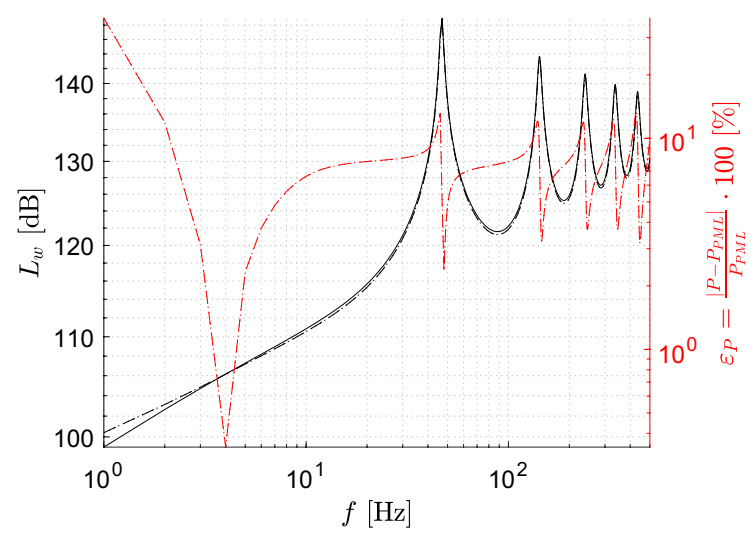

(b) $v_{s, \text { in }}=1 \mathrm{~ms}^{-1}$; geometry (b).

Fig. 9. (Color online) Sound power level $L_{w}$ and relative error $\varepsilon_{P}$ with respect to the PML solution for two different load cases and geometries. Geometry (a): error curves (pink), sound power curves (gray); geometry (b): error curves (red), sound power curves (black); PML reference curves (in Eqs. (5) and 17) ( - - - ), $P_{\mathrm{NM}}$ in Eq. (6) ( . . . ) .

\subsection{Modal sound power}

The two different load cases $v_{s, \text { out }}$ (for geometries (a) and (b)) and $v_{s, i n}$ (for geometry (b)) lead to the radiation of sound power, which is depicted in Fig. 9 in terms of the sound power level $L_{w}$. The sound power is determined by three different approaches for each problem: $P_{A, \text { inv }}$ - full inversion of the dynamic stiffness matrix for the vector of nodal sound pressure values in Eq. (7) to be substituted into Eq. (5), $P_{\mathrm{NM}}$ - full summation of modal sound power contributions according to Eq. (6) and $P_{\mathrm{PML}}$ - a reference solution is computed by using the commercial FE software COMSOL Multiphysics ${ }^{\circledR}$ and by applying PMLs.

It can be observed in Fig. 9(a) that the radiated sound power is almost identical for both geometries and approaches if the structural velocity $v_{s, \text { out }}$ is applied to the outer ellipsoidal surfaces of the obstacles. At the resonance frequencies of the cavity modes, the sound power level collapses slightly for geometry (b) with the opening, while the relative errors, with respect to the PML reference solution, increase significantly. However, the relative errors are less than $1 \%$ above $10 \mathrm{~Hz}$ and differ for both geometries, but do not for the different approaches, respectively.

If the velocity excitation is applied to the left end of the duct-like cavity (b), the resonances in the tube can clearly be found in the curves of the sound power levels (see Fig.9(b)). The relative errors of $P_{A \text {,inv }}$ and $P_{\mathrm{NM}}$ with respect to the PML solution are virtually the same and in the order of magnitude of $\approx 10 \%$ even though the approximation of the sound power seems to be reasonable in the whole frequency range.

Each NM has its own contribution to the total radiated sound pressure $\mathbf{p}_{m}$ and sound power $P_{m}$ (cf. Eqs. (4) and (6)). These two quantities are frequency-dependent and their modal contribution varies as the frequency changes. This is illustrated in Fig. 10(a) for $1 \mathrm{~Hz}$ and for multiple frequencies in Fig. 10(b) in the example of the second geometry and with 


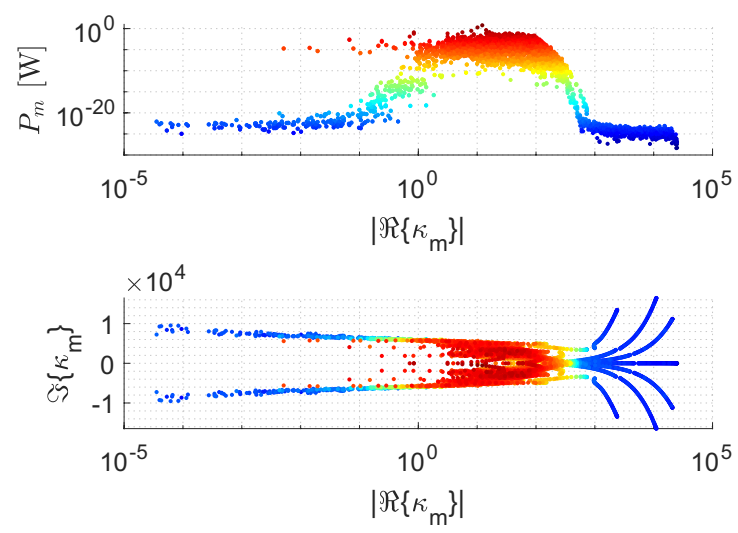

(a)

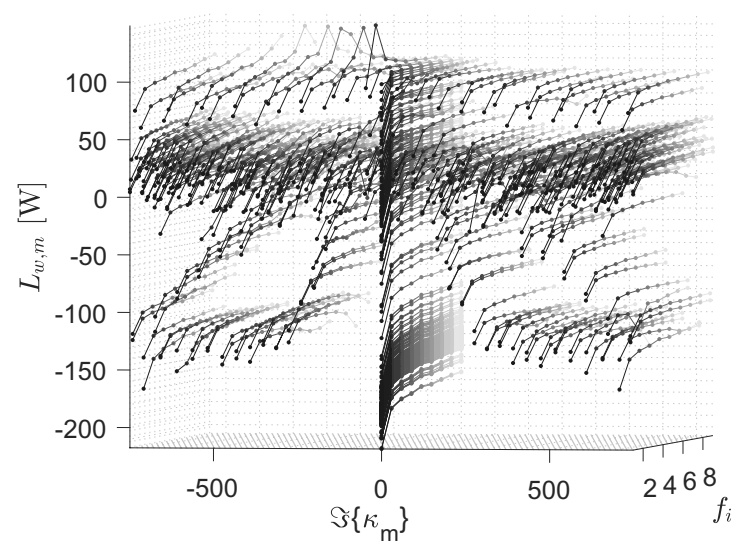

(b)

Fig. 10. (Color online) Modal sound power and sound power level contributions $P_{m}$ and $L_{w, m}$ of NM eigenvalues $m$ for geometry (b) and $v_{s, i n}$. (a) $P_{m}$ at $1 \mathrm{~Hz}$ in the third dimension of the complex plane (blue/small to red/high) and (b) $L_{w, m}$ at frequency steps $f_{i}$ (with increasing brightness) for $\Im\{\kappa\} \leq \mid \pm$ $750 \mathrm{~Hz} \mid$.

the excitation inside the open duct $v_{s, \text { in }}$. The largest sound power is contributed by NMs with a real part in a range of about 0.01 o 1000, which are most likely related to inner boundary modes according to Fig. Z7(b). At $1 \mathrm{~Hz}$, the sound power share in the complex plane is virtually symmetric with respect to the real axis (see Fig. 10(a)), i.e. the sound power contribution of the eigenvalues is almost the same for both complex conjugated partners. In Fig. 10(b), the sound power levels for nine frequency steps $f_{i}$ are only depicted for positive and negative imaginary parts of $\kappa_{m}$ that are smaller than $750 \mathrm{~Hz}$ according to the amount, for the sake of clarity. Five of these frequency steps $f_{i}$ are close to resonances of cavity modes. Most of the modal sound power contributions seem to increase as the frequency grows (which is emphasized by increasing the brightness of the lines), but only a few of them - primarily on the negative side of the imaginary axis - show clear peaks in particular at these resonance frequencies. This leads to an obviously asymmetric modal sound power level contribution for positive and negative imaginary parts of the complex eigenvalue partners. The differences of the modal sound power contributions of each of the two complex conjugated partners are depicted in Fig. 11, where the observations in Fig. 10(b) can be confirmed: The eigenvalue partners differ significantly at their corresponding cavity resonance frequencies only, where the radiation of sound is mainly due to the modes with the negative imaginary parts.

Different properties of the eigenvalues and eigenvectors have been discussed so far. With the aim of modal reduction during the summation process, a number of reduced modal bases is taken into account in Fig. 12, where the errors relative to $P_{\text {Ainv }}$ (Eqs. (5) and (7)) are depicted for both $v_{s, \text { out }}$ (Fig. 12(a)) and $v_{s, \text { in }}$ (Fig. 12(b)) for the ellipsoidal geometry with the cavity (b). The relative error for the full modal basis is almost equal to zero over the whole frequency range for both velocity excitations and shown as a red, thick 


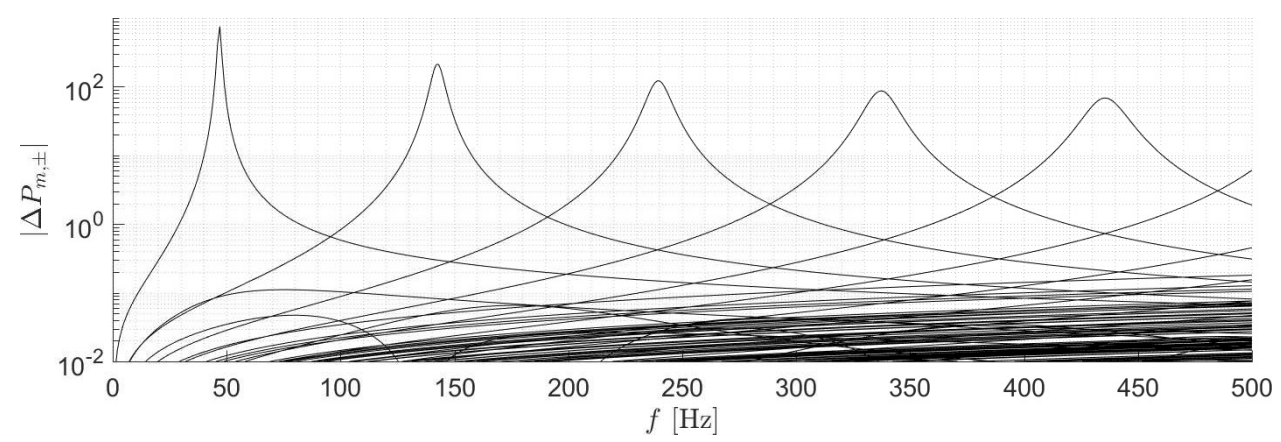

Fig. 11. Absolute sound power differences $\left|P_{m, \pm}\right|$ between 3316 complex conjugated eigenvalue partners $m$ as functions of the frequency for geometry (b) and $v_{s, \text { in }}$.

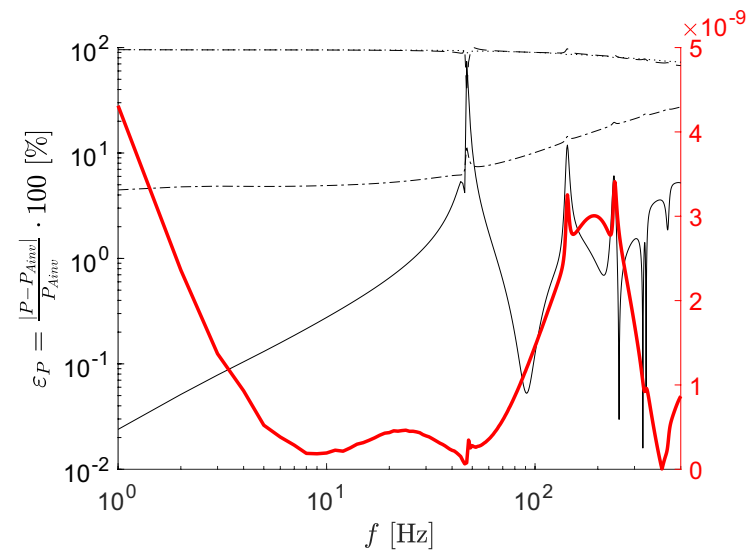

(a)

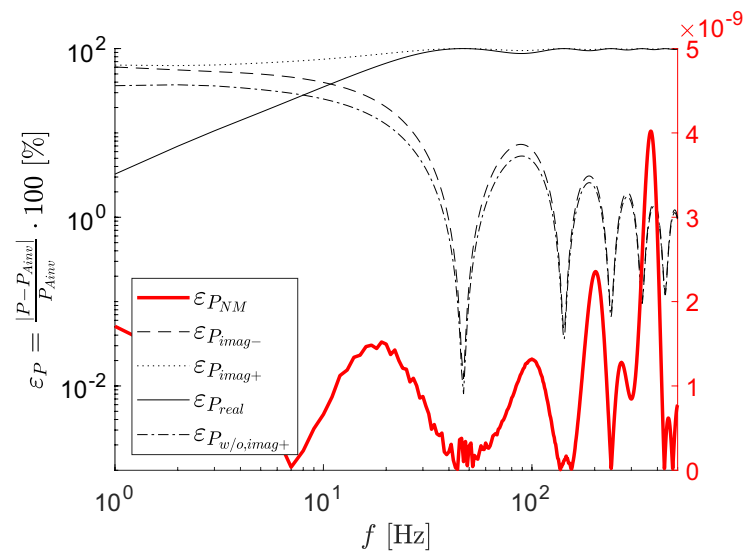

(b)

Fig. 12. (Color online) Errors of the superimposed sound power curves (relative to $P_{\text {Ainv }}$ ) for geometry (b) and both load cases $v_{s, \text { out }}$ and $v_{s, \text { in }}$ considering the whole modal basis (red, thick line), only modes with negative imaginary parts $(----)$, only modes with positive imaginary parts $(. .$.$) , only purely$ real modes (— $)$ and neglecting only the modes with positive imaginary parts $(-\cdot-\cdot-)$.

line, respectively. In the case of the outer excitation, the purely real eigenvalues lead to an acceptable relative error of less than $1 \%$, except in the region of the cavity resonance frequencies. When considering only complex eigenvalues with only positive or only negative imaginary parts, respectively, the relative errors are not acceptable for the given geometry and excitation. In the last case, only the complex eigenvalues with a positive imaginary part are removed from the whole modal basis, which leads to a better result over the whole frequency range and less significant error peaks at the resonances in particular. However, the overall error is much worse in comparison to the purely real modal basis.

If the velocity excitation is applied inside the open duct, the purely real modal basis performs almost as poorly as the complex eigenvalues with a positive imaginary part, as can be seen in Fig. 12(b). The relative error is acceptable for frequencies higher than $10 \mathrm{~Hz}$ for 


\section{Moheit \&3 S. Marburg}

the two other methods: the eigenvalues with negative imaginary parts and — even better the modal basis without the complex eigenvalues with a positive imaginary part.

Altogether, this leads to the assumption that pure exterior problems are mainly dominated by the purely real eigenvalues, and the cavity modes are due to the complex eigenvalues with a negative imaginary part. Neglecting the eigenvalues with a positive imaginary part only leads to acceptable results in the given example. However, better performance is desired and the criteria for reduced modal bases have to be further developed.

\section{Conclusion}

The sound pressure and sound power due to structural velocities in exterior problems could be determined by superposition of NMs. The major part of the eigenvalues is not problemspecific and due to the FE and IFE mesh. It was found that for weakly damped modes, the sound pressure is mainly concentrated at the surfaces of inner obstacles in the fluid, whereas highly damped modes have a large pressure distribution at the junction of the FE and the IFE domains. However, the largest share of radiated sound power is not due to the modes with the smallest damping coefficient, but it is mainly dominated by cavity resonances. The authors observed that the sound power peaks at these resonances are due to modes with a negative imaginary part, whereas the positive complex conjugated partners play a minor role in the radiation of sound. In order to further reduce the modal basis and - at the same time - minimize the errors during the modal superposition, additional criteria have to be developed in future work.

\section{Acknowledgments}

The support by the Deutsche Forschungsgemeinschaft (DFG project number 230029255) is gratefully acknowledged by the authors.

\section{References}

1. S. H. Schot, Eighty years of Sommerfeld's radiation condition, Historia Math. 19(4) (1992) 385-401.

2. O. C. Zienkiewicz and R. L. Taylor, The Finite Element Method for Solid and Structural Mechanics (Butterworth-Heinemann, Oxford, 2005).

3. D. Givoli, T. Hagstrom and I. Patlashenko, Finite element formulation with high-order absorbing boundary conditions for time-dependent waves, Comput. Methods Appl. Mech. Eng. 195(29) (2006) 3666-3690.

4. D. Givoli, Computational absorbing boundaries, in Computational Acoustics of Noise Propagation in Fluids - Finite and Boundary Element Methods (Springer, Berlin, 2008), pp. 145-166.

5. D. Rabinovich, D. Givoli and E. Bécache, Comparison of high-order absorbing boundary conditions and perfectly matched layers in the frequency domain, Int. J. Numer. Methods Biomed. Eng. 26(10) (2010) 1351-1369.

6. J.-P. Berenger, A perfectly matched layer for the absorption of electromagnetic waves, J. Comput. Phys. 114(2) (1994) 185-200. 
7. A. Bermúdez, L. Hervella-Nieto, A. Prieto and R. Rodríguez, Perfectly matched layers, in Computational Acoustics of Noise Propagation in Fluids - Finite and Boundary Element Methods, eds. S. Marburg and B. Nolte (Springer, Berlin, Heidelberg, 2008), pp. 167-196.

8. A. Bermúdez, L. Hervella-Nieto, A. Prieto and R. Rodríguez, Perfectly matched layers for timeharmonic second order elliptic problems, Arch. Comput. Methods Eng. 17(1) (2010) 77-107.

9. P. Bettess, Infinite elements, Int. J. Numer. Methods Eng. 11(1) (1977) 53-64.

10. D. S. Burnett, A three-dimensional acoustic infinite element based on a prolate spheroidal multipole expansion, J. Acoust. Soc. Am. 96(5) (1994) 2798-2816.

11. R. J. Astley, Infinite elements, in Computational Acoustics of Noise Propagation in Fluids Finite and Boundary Element Methods, eds. S. Marburg and B. Nolte (Springer, Berlin, Heidelberg, 2008), pp. 197-230.

12. R. J. Astley, G. J. Macaulay, J.-P. Coyette and L. Cremers, Three-dimensional wave-envelope elements of variable order for acoustic radiation and scattering. Part i. Formulation in the frequency domain, J. Acoust. Soc. Am. 103(1) (1998) 49-63.

13. R. J. Astley and J.-P. Coyette, Conditioning of infinite element schemes for wave problems, Commun. Numer. Methods Eng. 17(1) (2001) 31-41.

14. O. von Estorff, S. Petersen and D. Dreyer, Efficient infinite elements based on Jacobi polynomials, in Computational Acoustics of Noise Propagation in Fluids - Finite and Boundary Element Methods, eds. S. Marburg and B. Nolte (Springer, Berlin, Heidelberg, 2008), pp. 231-250.

15. D. Dreyer and O. von Estorff, Improved conditioning of infinite elements for exterior acoustics, Int. J. Numer. Methods Eng. 58(6) (2003) 933-953.

16. T. W. Wu, Boundary Element Acoustics Fundamentals and Computer Codes (WIT Press, Southampton, 2000).

17. S. Marburg and B. Nolte, A unified approach to finite and boundary element discretization in linear time-harmonic acoustics, in Computational Acoustics of Noise Propagation in Fluids Finite and Boundary Element Methods, eds. S. Marburg and B. Nolte (Springer, Berlin Heidelberg, 2008), pp. 1-34.

18. S. Marburg, Normal modes in external acoustics. Part i: Investigation of the one-dimensional duct problem, Acta Acust. United Ac. 91(6) (2005) 1063-1078.

19. S. Marburg, F. Dienerowitz, T. Horst and S. Schneider, Normal modes in external acoustics. part ii: Eigenvalues and eigenvectors in 2d, Acta Acust. United Ac. 92(1) (2006) 97-111.

20. S. Marburg, Normal modes in external acoustics. Part iii: Sound power evaluation based on superposition of frequency-independent modes, Acta Acust. United Ac. 92(2) (2006) 296-311.

21. L. Moheit and S. Marburg, Infinite elements and their influence on normal and radiation modes in exterior acoustics, J. Comput. Acoust. 25(04) (2017) 1650020.

22. L. Moheit and S. Marburg, Acoustic radiation modes and normal modes in unbounded domains, in Proc. Meetings on Acoustics 173EAA (ASA Conference, Boston, MA USA, 2017), p. 022004.

23. P. Ruge, Eigenvalues of damped structures: Vectoriteration in the original space of dof, Comput. Mech. 22(2) (1998) 167-173.

24. F. Tisseur and K. Meerbergen, The quadratic eigenvalue problem, SIAM Rev. 43(2) (2001) 235-286.

25. S. Fuß, S. C. Hawkins and S. Marburg, An eigenvalue search algorithm for the modal analysis of a resonator in free space, J. Comput. Acoust. 19(01) (2011) 95-109.

26. A. Kuijpers, G. Verbeek and J. Verheij, An improved acoustic fourier boundary element method formulation using fast fourier transform integration, J. Acoust. Soc. Am. 102(3) (1997) 13941401.

27. P. H. L. Kessels, Engineering Toolbox for Structural-acoustic Design: Applied to MRI-Scanners (Technische Universiteit Eindhoven, Netherlands, 2001). 
28. H. Peters, N. Kessissoglou and S. Marburg, Enforcing reciprocity in numerical analysis of acoustic radiation modes and sound power evaluation, J. Comput. Acoust. 20(03) (2012) 1250005.

29. H. Peters, N. Kessissoglou and S. Marburg, Modal decomposition of exterior acoustic-structure interaction, J. Acoust. Soc. Am. 133(5) (2013) 2668-2677.

30. H. Peters, N. Kessissoglou and S. Marburg, Modal decomposition of exterior acoustic-structure interaction problems with model order reduction, J. Acoust. Soc. Am. 135(5) (2014) 2706-2717.

31. J. Baumgart, S. Marburg and S. Schneider, Efficient sound power computation of open structures with infinite/finite elements and by means of the Padé-via-Lanczos algorithm, J. Comput. Acoust. 15(04) (2007) 557-577.

32. M. M. Wagner, P. M. Pinsky, A. A. Oberai and M. Malhotra, A Krylov subspace projection method for simultaneous solution of Helmholtz problems at multiple frequencies, Comput. Methods Appl. Mech. Eng. 192(41) (2003) 4609-4640.

33. S. Marburg, Six boundary elements per wavelength: Is that enough? J. Comput. Acoust. 10(01) (2002) 25-51.

34. P. Langer, M. Maeder, C. Guist, M. Krause and S. Marburg, More than six elements per wavelength: The practical use of structural finite element models and their accuracy in comparison with experimental results, J. Comput. Acoust. 25 (2017) 1750025.

35. R. J. Allemang, The modal assurance criterion-twenty years of use and abuse, J. Sound Vib. 37(8) (2003) 14-23. 